\title{
Internal energy selection in vacuum ultraviolet photoionization of ethanol and ethanol dimers
}

\author{
Andras Bodia) \\ Molecular Dynamics Group, Paul Scherrer Institut, 5232 Villigen, Switzerland
}

(Received 21 August 2013; accepted 19 September 2013; published online 10 October 2013)

\begin{abstract}
Internal energy selected ethanol monomer and ethanol dimer ions were prepared by threshold photoionization of a supersonic molecular beam seeded with ethanol. The dissociative photoionization processes of the monomer, the lowest-energy $\mathrm{CH}_{3}$-loss channel of the dimer, and the fragmentation of larger clusters were found to be disjunct from the ionization onset to about $12 \mathrm{eV}$, which made it possible to determine the $0 \mathrm{~K}$ appearance energy of $\mathrm{C}-\mathrm{C}$ bond breaking in the $\mathrm{H}$-donor unit of the ethanol dimer cation as $9.719 \pm 0.004 \mathrm{eV}$. This reaction energy is used together with $a b$ initio calculations in a thermochemical cycle to determine the binding energy change from the neutral ethanol dimer to a protonated ethanol-formaldehyde adduct. The cycle also shows general agreement between experiment, theory, and previously published enthalpies of formation. The role of the initial ionization site, or rather the initial photoion state, is also discussed based on the dimer breakdown diagram and excited state calculations. There is no evidence for isolated state behavior, and the ethanol dimer dissociative photoionization processes appear to be governed by statistical theory and the ground electronic state of the ion. In the monomer breakdown diagram, the smoothly changing branching ratio between $\mathrm{H}$ and $\mathrm{CH}_{3}$ loss is at odds with rate theory predictions, and shows that none of the currently employed few-parameter rate models, appropriate for experimental rate curve fitting, yields a correct description for this process in the experimental energy range. (C) 2013 AIP Publishing LLC. [http://dx.doi.org/10.1063/1.4824135]
\end{abstract}

\section{INTRODUCTION}

Photoionization mass spectrometry (PIMS) is a selective and sensitive detection method, which, combined with tunable synchrotron vacuum ultraviolet (VUV) radiation, often allows for isomer specific detection of reaction intermediates in complex chemical systems. ${ }^{1}$ It has also been used for several decades to derive appearance potentials, which frequently correspond to dissociative photoionization energies and can be used in thermochemical derivations. ${ }^{2,3}$ Interpreting photoionization efficiency curves is, however, a delicate art, even when the unimolecular dissociation reaction is well understood. ${ }^{4}$ Photoion internal energy selection in photoelectron photoion coincidence (PEPICO) spectroscopy takes us one step closer to the complete experiment, by controlling how much internal energy is deposited in the parent ion. ${ }^{5}$ In threshold (T)PEPICO, close to zero kinetic energy electrons are selected for and act as start signal for the ion time-offlight (TOF) analysis. Additionally, low photoion draw out fields and long acceleration regions can lead to long photoion residence times, which enables dissociation rate measurements, and a quantitative treatment of kinetic shifts in a statistical model. A dissociation rate curve can be constructed based on the measured unimolecular dissociation rates and extrapolated to threshold. ${ }^{6}$

Even though homolytic bond cleavage reactions in cations typically proceed along purely attractive potential energy curves, dissociative photoionization onsets still have to

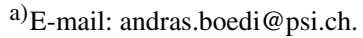

be interpreted cautiously even when measured in TPEPICO experiments. Hydrogen atom loss from formic acid cations, for example, involves a reverse barrier despite the absence of obvious rearrangements along the reaction coordinate. ${ }^{7}$ The lowest energy dissociative photoionization channel of methanol is $\mathrm{H}$ loss, the appearance energy of which was used to derive the most accurate experimental proton affinity of formaldehyde. ${ }^{8}$ The same approach failed for the analogous $\mathrm{H}$ loss in ethanol cations, which takes place above and across a reverse barrier. ${ }^{9}$ The latter work supplied tunneling data for light and deuterated ethanol isotopologues reassuringly congruent with quantum chemical calculations, but it also left the question of modeling the second lowest energy channel, methyl loss, partially open. Two rate models have recently been used to account for kinetic shifts: the Rice-RamspergerKassel-Marcus (RRKM) model has been shown to overestimate the kinetic shift in halobenzenes, ${ }^{10}$ and the simplified statistical adiabatic channel model $(\mathrm{SSACM})^{11}$ was proposed instead to fit experimental rate curves of unimolecular reactions without a true transition state, i.e., without a reverse barrier. The RRKM model predicts that methyl loss overtakes hydrogen atom loss quickly and quantitatively in the dissociative photoionization of ethanol, because the transition state of the former is much looser. The experiment showed, however, this not to be the case. ${ }^{9}$ SSACM can be considered as a first order correction to phase space theory, ${ }^{12,13}$ i.e., it puts the transition state at the products, and has been successfully applied to model the rising edge of the methyl-loss signal.

The fractional abundances of the $\mathrm{CH}_{2} \mathrm{OH}^{+}$and $\mathrm{CH}_{3} \mathrm{CHOH}^{+}$fragment ions as a function of the $\mathrm{C}_{2} \mathrm{H}_{5} \mathrm{OH}^{+}$ 
internal energy, i.e., the photon energy, in the breakdown diagram are determined by the branching ratio, in other words by the ratio of the dissociation rate constants. The applicability of the RRKM model has thus been ruled out, because $\mathrm{CH}_{3}$ loss does not outcompete $\mathrm{H}$ loss immediately above threshold. The methyl-loss product ion, $\mathrm{CH}_{2} \mathrm{OH}^{+}$, has a low density of states with the lowest energy vibration at more than $1000 \mathrm{~cm}^{-1}$, similarly to $\mathrm{CH}_{3}$, which has the lowest energy vibrational transition at about $500 \mathrm{~cm}^{-1}$. Because of the tight product ions in $\mathrm{CH}_{3}$-loss and the smoothly changing $\mathrm{H}$-loss rates, the branching ratios were predicted to be oscillatory above the crossover energy of $\mathrm{H}$ and $\mathrm{CH}_{3}$ loss by SSACM. This effect could not be confirmed or dismissed based on room temperature data. The motivation of the present work is in part to report branching ratios for the dissociative photoionization of cold ethanol to enhance the predicted oscillatory behavior and lower its detection limit.

Furthermore, a photoionization mass spectrometry study has recently been published on the site selective ionization of ethanol dimers. ${ }^{14}$ The detailed mechanism of (threshold) photoionization and subsequent dissociation in different experimental techniques is an intriguing question. ${ }^{15,16}$ Breakdown diagrams and dissociation rates curves have been modeled consistently for over a hundred systems, ${ }^{6}$ and the overwhelming majority was found to behave statistically in valence photoionization. Typically, threshold photoionization cross-sections are constant over the internal energy distribution of the sample, from which it follows that the shape of the internal energy distribution is conserved and is only shifted onto the ion manifold. Thereafter, the plethora of conical intersections available on the ion potential energy surface allows for internal conversion to the ground electronic state. Since the phase space of the parent ion is dominated by the ground electronic state at any internal energy, it alone will shape the dissociation mechanism of the photoion.

We have previously argued that even though nonstatistical dissociation dynamics has often been invoked either to explain discrepancies between experiment and theory or to offer an appealing dissociation model, such proposals need to go hand in hand with a profound understanding of the fate of the parent ion, and when and how different electronic or nuclear degrees of freedom are frozen along the dissociation coordinate. ${ }^{17}$ To block access to parts of the phase space and thereby breach the ergodic hypothesis, the dissociation process has to take place on a faster time scale than the statistical redistribution of internal energy among the different degrees of freedom, i.e., on a non-ergodicity time scale. ${ }^{18} \mathrm{In}$ tetrafluoroethene, for example, a broad Franck-Condon gap between the first electronically excited $\tilde{A}$ and ground $\tilde{X}$ ion states results in a lack of intermediate states and blocks internal conversion to the ground state. ${ }^{19}$ Consequently, excited $\mathrm{C}_{2} \mathrm{~F}_{4}{ }^{+}$ions establish a second dissociation regime, which can be modeled statistically but for the electronic degree of freedom. A different picture emerged for monofluoroethene, in which the internal conversion path from the $\tilde{C}$ to the $\tilde{X}$ state crosses the $\tilde{B}$ state in two domains, in which the $\tilde{B}$ state can have either a bound or a repulsive character. ${ }^{20}$ Thereby, the latter conical intersection opens up a non-statistical Floss channel. A further distinct phenomenon is when the
Franck-Condon factors are negligible in the potential energy well of the parent ion, and the large force along the reaction coordinate in the Franck-Condon envelope leads to an impulsive dissociation, as is the case in the ground electronic state of $\mathrm{CF}_{4}{ }^{+}$and $\mathrm{CCl}_{4}{ }^{+} .{ }^{21,22}$

Another possibility, perhaps more relevant to the study of the dissociative photoionization of clusters, is that the timescale of intramolecular vibrational relaxation (IVR) outgrows the timescale of dissociation. Since the engine of IVR is anharmonicity, it will slow down in large molecules or in loosely bound adducts, in which the average energy and thereby the anharmonicity in each degree of freedom is low at the dissociation threshold. ${ }^{23-26}$ It is therefore interesting to compare the PIMS results on the ethanol dimer with the threshold photoionization and discuss how to interpret site-selective ionization in this process.

\section{EXPERIMENTAL AND COMPUTATIONAL METHODS}

Imaging photoelectron photoion coincidence (iPEPICO) experiments were carried out at the VUV beamline of the Swiss Light Source. ${ }^{27-29}$ Slightly more than 1 bar of Ar was saturated with ethanol in a bubbler at room temperature, and its backing pressure regulated to $0.40 \mathrm{bar}$ with a needle valve. It expanded through a $50 \mu \mathrm{m}$ nozzle into the source chamber, where the pressure was close to $10^{-4}$ mbar. The molecular beam was skimmed in the experimental chamber to a background pressure of $2.5 \times 10^{-6}$ mbar. In the iPEPICO experimental chamber, the sample is photoionized by the incident monochromatic VUV radiation with a $2-4 \mathrm{meV}$ resolution and higher harmonics suppressed with a differentially pumped gas filter using a Kr:Ne:Ar mixture. The photoelectrons are velocity map imaged onto a fast delay-line detector and also act as start-signal for the photoion time-of-flight analysis. The kinetic energy electron contamination of the central, threshold spot on the electron velocity map image is subtracted to obtain threshold photoionization mass spectra ${ }^{30}$ in the 9.5-14 eV photon energy range with integration times ranging from $180 \mathrm{~s}$ to $1200 \mathrm{~s}$.

In the iPEPICO experiment, asymmetric fragment ion TOF distributions are indicative of slow dissociations and are a direct experimental measure of the dissociation rates. ${ }^{6}$ When the TOF difference between the parent and fragment ion is small, however, and the peak broadening becomes commensurate with the instrumental peak width, the daughter ion center of gravity $(\mathrm{CoG})$ can be used as an experimental measure of the dissociation rate ${ }^{31}$ or, together with higher moments of the TOF band, even to deconvolute more peaks in a TOF band. ${ }^{32}$ Within the signal-to-noise ratio, the CoG of all major peaks were constant with the exception of a slight asymmetry of the protonated trimer peak $(\mathrm{m} / \mathrm{z}=139)$, which may indicate that $\mathrm{C}_{2} \mathrm{H}_{5} \mathrm{O}$ loss from the tetramer is barely metastable $\left(k \approx 10^{7} \mathrm{~s}^{-1}\right)$ at threshold, as well as the H-loss peak from $\mathrm{C}_{2} \mathrm{H}_{5} \mathrm{OH}^{+}$, which was shown to be metastable due to tunneling through a reverse barrier. ${ }^{9}$ No other parent ion is metastable on the time scale of the experiment, and the instrumental peak width does not hide changing $\mathrm{m} / \mathrm{z}$ vs. $\mathrm{m} / \mathrm{z}-1$ peak ratios at higher masses. 


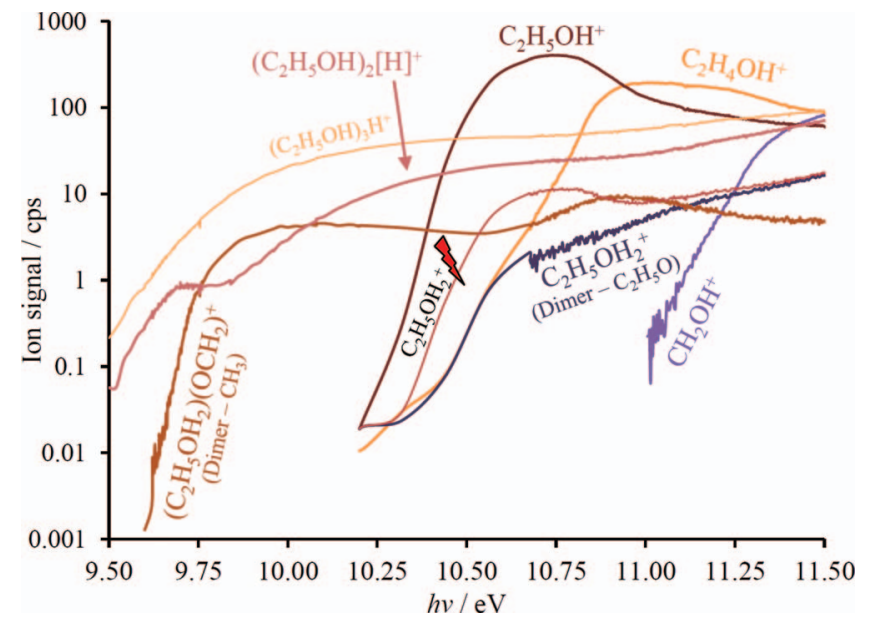

FIG. 1. Ion signal as a function of photon energy in coincidence with all recorded electrons. The lightning bolt highlights the $m / z=47 \mathrm{C}_{2} \mathrm{H}_{5} \mathrm{OH}_{2}{ }^{+}$ curve, from which the ${ }^{13} \mathrm{C}$ contribution of the $\mathrm{m} / z=46$ ethanol signal has not been subtracted.

Quite often, experimental dissociative photoionization data can only be meaningfully interpreted with the help of $a b$ initio calculations supporting the purported dissociation mechanism and energetics. To this end, CBS-APNO and W1U composite and M06-2X/6-311++G(d,p) density functional theory as well as M06-2X/aug-cc-pVTZ timedependent density function theory calculations were carried out using the Gaussian09 program package. ${ }^{33-36}$ Ion electronic states and adiabatic and vertical ionization energies were also computed with the equation-of-motion coupled-cluster singles and doubles for ionization potential (EOM-IP-CCSD) method ${ }^{37,38}$ and the cc-pVDZ and cc-pVTZ basis sets using Q-Chem 4.1.39,40

\section{RESULTS AND DISCUSSION}

The total recorded ion signal is shown in Figure 1, a logarithmic scale, because it covers six orders of magnitude. The maximum kinetic energy of the electrons, which are still completely imaged onto the detector is about $1 \mathrm{eV}$, but in order to save disk space, electrons above a radius corresponding to $100 \mathrm{meV}$ were discarded. This means that the electron collection efficiency decays reciprocally as $0.1 \mathrm{eV} / \mathrm{E}$ above a kinetic energy of $0.1 \mathrm{eV}$. The plotted curves are, thus, expected to agree with the photoionization efficiency (PIE) curves obtained in PIMS as reported by Li et al. ${ }^{14}$ close to the ionization onsets of the ions and slowly turn into PIE curve derivatives as the photon energy increases significantly above $0.1 \mathrm{eV}$ from the onset. Protonated cluster signal has been observed up to $n=7$, which mirror that of the protonated trimer only with a lower onset energy and are not plotted. As will be shown later, this plot only conveys a fraction of the experimental information obtained in threshold photoionization. The dimer and protonated dimer signals have not been deconvoluted and their sum has a plateau at a photon energy of about $9.75 \mathrm{eV}$, whereas the monomer signal effectively drops above $10.75 \mathrm{eV}$, which correspond to the opening up of the $\mathrm{CH}_{3}$-loss and $\mathrm{H}$-loss dissociative photoionization channels in the dimer and monomer, respectively. Li et al. report the appearance of the $m / z=47 \mathrm{C}_{2} \mathrm{H}_{5} \mathrm{O}$-loss fragment ion from the dimer, $\mathrm{C}_{2} \mathrm{H}_{5} \mathrm{OH}_{2}{ }^{+}$at its calculated thermochemical threshold, $10.25 \pm 0.05 \mathrm{eV}, 100 \mathrm{meV}$ below the computed result reported herein (see later). Even at $10.44 \mathrm{eV}$ photon energy, $1000 \mathrm{~s}$ integration time, and $\mathrm{C}_{2} \mathrm{H}_{5} \mathrm{OH}^{+}$count of more than 20000 , the $m / z=47$ channel consists, within the experimental uncertainty, entirely of the $2.3 \%{ }^{13} \mathrm{C} /{ }^{2} \mathrm{H}$ peak of the $m / z=46$ ethanol signal. The ethanol monomer ionization onset of $10.47 \pm 0.05 \mathrm{eV}$, reported by $\mathrm{Li}$ et al. and based on a linear fit to the PIE curve, is $0.1 \mathrm{eV}$ above the adiabatic ionization energy, ${ }^{9}$ and corresponds to an ethanol cation signal of $\approx 45$ counts/s in our experiment. The $\mathrm{C}_{2} \mathrm{H}_{5} \mathrm{OH}_{2}{ }^{+}$ signal level at a photon energy of $10.25 \mathrm{eV}$ is less than 0.1 counts/s in our experiment, so the Li et al. onset is either based on an extrapolation of the ethanol ${ }^{13} \mathrm{C}$ peak or on a different approach to determining PIE onsets than employed in the ethanol monomer. Since Franck-Condon factors are unfavorable for adiabatic ionization in ethanol monomer, dimer, and larger clusters, the experimental parent ion onset energies are solely indicative of instrument sensitivity and do not correspond to a physical quantity.

The threshold photoionization yield can be obtained by discriminating for close to zero kinetic energy electrons as start signals, focused onto the center of the velocity map image and subtracting the kinetic energy contamination. ${ }^{30}$ As shown in Figure 2, threshold photoionization data are informative, because they clearly show the course of dissociative
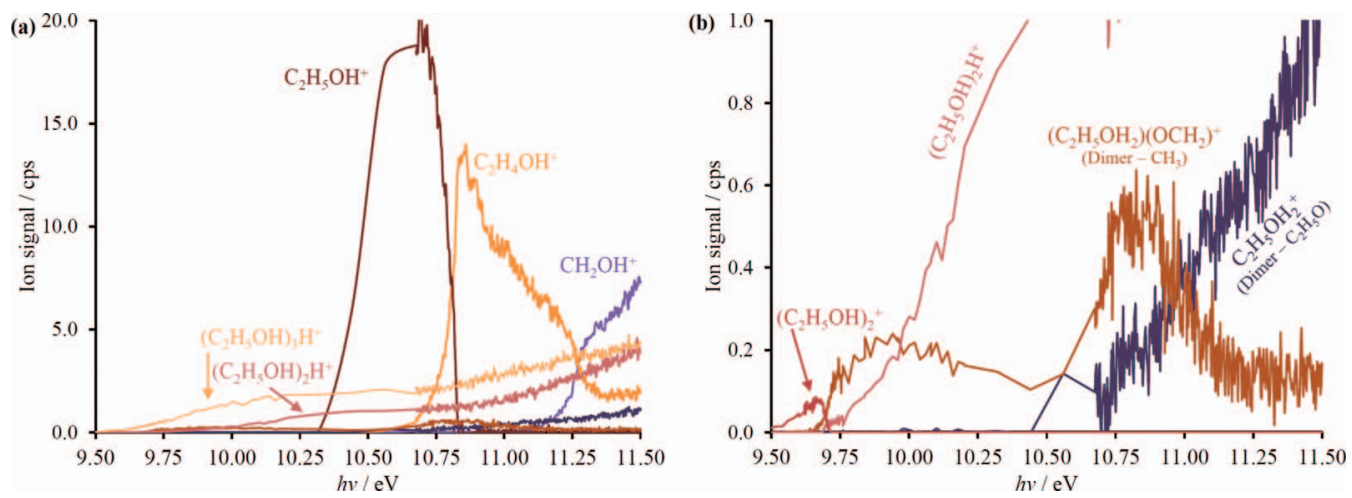

FIG. 2. Threshold photoionization efficiency curves as a function of photon energy. The overall signal in (a) is dominated by the monomer contribution, whereas the low-energy cluster data correspond to ionization yields of less than 1 counts/s in (b). 
photoionization processes and allow for the construction of threshold photoionization breakdown diagrams. ${ }^{5}$ The monomer ethanol cation dominates the signal in Figure 2(a), with protonated trimer and dimer ion yields becoming commensurate above $11 \mathrm{eV}$. This may be the combined result of higher clusters cascading down to lower ones at such internal energies, and, to a lesser extent, the more favorable Franck-Condon factors to produce electronically excited cluster ion states. In the internal energy selected threshold photoionization picture of the dimer ion in Figure 2(b), the intact dimer is first observed to lose a methyl group and disappears within $50 \mathrm{meV}$. Thereafter, the methyl-loss signal from the dimer ion yields slowly, over $500 \mathrm{meV}$ to the $\mathrm{C}_{2} \mathrm{H}_{5} \mathrm{O}$-loss ion $\mathrm{C}_{2} \mathrm{H}_{5} \mathrm{OH}_{2}{ }^{+}$in the $10.75-11.25 \mathrm{eV}$ photon energy range.

What do these threshold photoionization ion yields tell us about the dissociative photoionization mechanism? First, the ethanol monomer ion disappears quantitatively above the dissociative photoionization onset of H-loss. ${ }^{9}$ If ethanol ions were also made by the dissociative photoionization of clusters, they would lose some of the excess energy to the leaving neutral fragment and through kinetic energy release, meaning that they could only lose an $\mathrm{H}$ atom at higher photon energies than the monomer onset. Thus, contrary to the assumptions based on the HOMO-1 orbital character in the ethanol dimer, ${ }^{14}$ the dissociative photoionization of the dimer or larger clusters does not appear to yield monomer cations in the energy range studied. Second, the sudden disappearance of the intact dimer ion signal at $9.72 \mathrm{eV}$ tells us a similar story on dimer cations, namely that the dissociative photoionization of larger cluster ions does not yield ethanol dimer fragment ions. The sharp crossover between the dimer ion and the methyl-loss fragment ion at $9.70 \mathrm{eV}$ without any daughter ion asymmetry implies that all dimer ions with sufficient internal energy to dissociate will also do so. Thus, the dissociative photoionization of larger ethanol clusters can only yield protonated ethanol clusters. The threshold photoionization curves of the protonated cluster ions do not exhibit clear crossover ranges, which is not unexpected in the light of the numerous dissociation pathways available to larger clusters. At the same time, the dimers remain conveniently detached from the manifold of protonated clusters allowing for their detailed analysis. However, the lingering $\left(\mathrm{C}_{2} \mathrm{H}_{5} \mathrm{OH}_{2}\right)\left(\mathrm{OCH}_{2}\right)^{+}$

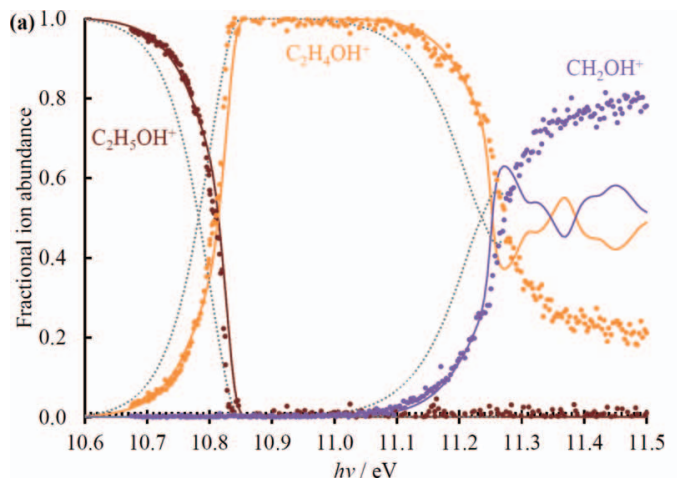

signal and the rising protonated monomer signal above $11.5 \mathrm{eV}$ may be a result of the presence of higher clusters in the beam. However, their dissociative photoionization or intracluster reactions ${ }^{41}$ do not affect the data analysis of the dimer data.

Third, if the $\mathrm{C}_{2} \mathrm{H}_{5} \mathrm{O}$ loss were related to the ionization site, i.e., there were some non-statistical character to $\mathrm{C}_{2} \mathrm{H}_{5} \mathrm{O}$ loss, the fragment ion yield curve would be expected to correlate with the threshold photoionization cross sections, with its rise perhaps preceding the isolated electronic state because of Rydberg-state involvement. ${ }^{20}$ This is clearly not the case here, as the branching ratio between $\mathrm{C}_{2} \mathrm{H}_{5} \mathrm{O}$ loss and $\mathrm{CH}_{3}$ loss changes slowly with the photon energy past the onset of the $\tilde{A}$ state of the dimer at about $10.5 \mathrm{eV}$. Such slowly changing branching ratios are characteristic of parallel processes, ${ }^{42}$ which means that both dissociation reactions take place on the ground electronic state potential energy surface. At the same time, $\mathrm{C}_{2} \mathrm{H}_{5} \mathrm{O}$ loss outcompetes $\mathrm{CH}_{3}$ loss already at $11.25 \mathrm{eV}$. The question whether $\left(\mathrm{C}_{2} \mathrm{H}_{5} \mathrm{OH}_{2}\right)\left(\mathrm{OCH}_{2}\right)^{+}$or the less stable $\left(\mathrm{C}_{2} \mathrm{H}_{5} \mathrm{OH}\right)\left(\mathrm{CH}_{2} \mathrm{OH}\right)^{+}$is formed by methyl loss after dissociative photoionization from an originally $\tilde{A}$ state dimer ion would only be relevant if significant methyl loss production was observed in this energy range. In fact, the methylloss fragment ion signal stays constant at 0.13 counts/s from $11.25 \mathrm{eV}$ to $13 \mathrm{eV}$, making it unlikely that a new fragmentation channel opens up below $13 \mathrm{eV}$. In short, the internal energy selection in threshold photoionization ion yield curves, shown in Figure 2, helps establish that ethanol dimer ions behave statistically, and the initial ion state or ionization site does not influence the mechanism, as the parent ion is long-lived for the different electronic and nuclear degrees of freedom to mix.

The data analysis can be completed based on the breakdown diagrams, plotted for ethanol in Figure 3(a). Ethanol cations lose $\mathrm{H}$ across a reverse barrier and $\mathrm{CH}_{3}$ in a parallel process at higher energies. Data for the ethanol dimer are shown in Figure 3(b), which loses $\mathrm{CH}_{3}$ in the lowest energy dissociative photoionization channel, followed by a parallel, $\mathrm{C}_{2} \mathrm{H}_{5} \mathrm{O}$-loss channel. The breakdown diagram model for the room temperature ethanol experiment is also plotted in Figure 3(a) for comparison. ${ }^{9}$ The appearance of both $\mathrm{H}$-loss and $\mathrm{CH}_{3}$-loss fragment ions is quite well reproduced by a linear combination of the room temperature

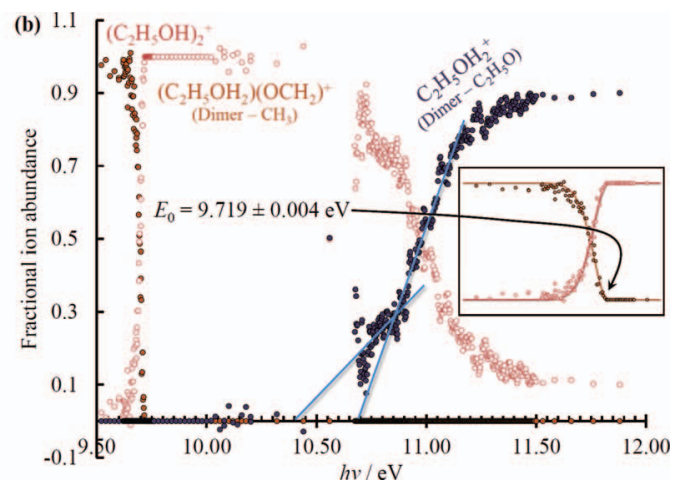

FIG. 3. Breakdown diagrams for (a) ethanol and (b) ethanol dimer. Experimental points are dots and the statistical model curves are plotted as continuous lines. The room temperature ethanol breakdown diagram model is shown in (a) as dotted lines for comparison. The inset in (b) shows the model for $\mathrm{CH}_{3}$ loss from the dimer ion in the $9.52-7.78 \mathrm{eV}$ photon energy range. 
and a $70 \mathrm{~K}$ model with equal weights, similarly to what was observed for propane. ${ }^{43}$

The ethanol parent ion disappears from the breakdown diagram at marginally lower photon energy than modeled, which could be a result of incomplete cooling and the resulting non-Boltzmann type internal energy distribution. On the other hand, the oscillations predicted by phase space theory due to the tightness of the protonated formaldehyde, $\mathrm{CH}_{2} \mathrm{OH}^{+}$, and $\mathrm{CH}_{3}$ products in the second step are enhanced by the model at low temperatures and clearly absent in the experiment. The RRKM rate theory has been shown to fail for this dissociation, ${ }^{9}$ and the SSACM approach is, apparently, also only applicable close to the onset energy. The significant thermal background in the molecular beam experiment could be quantitatively suppressed in an ion imaging experiment, ${ }^{29}$ which would make it worthwhile to apply more rigorous rate theories to try to model the branching ratio of these two parallel channels as a function of internal energy. At the same time, the quantitative disappearance of the monomer ethanol signal above the $\mathrm{H}$-loss onset and the well reproduced onset of the $\mathrm{CH}_{3}$-loss product ion breakdown curve verify that the monomer breakdown diagram is unaffected by the dissociative photoionization of dimers or larger clusters.

The overall appearance of the dimer breakdown diagram with two parallel processes as shown in Figure 3(b) is quite like that of the monomer. The lowest energy channel in the dimer, methyl radical loss, is the higher energy process in the monomer breakdown diagram. As shown in the inset, this process can be modeled assuming an $80 \mathrm{~K}$ internal energy distribution of the ethanol dimers and a fast dissociation. ${ }^{6}$ The resulting $0 \mathrm{~K}$ appearance energy of $9.719 \pm 0.004 \mathrm{eV}$, i.e., $937.7 \pm 0.4 \mathrm{~kJ} \mathrm{~mol}^{-1}$, corresponds to the $0 \mathrm{~K}$ reaction energy of $\left(\mathrm{C}_{2} \mathrm{H}_{5} \mathrm{OH}\right)_{2} \rightarrow\left(\mathrm{C}_{2} \mathrm{H}_{5} \mathrm{OH}_{2}\right)\left(\mathrm{OCH}_{2}\right)^{+}+\mathrm{CH}_{3}$ $+e^{-}$. The appearance energy range of the $\mathrm{C}_{2} \mathrm{H}_{5} \mathrm{O}$-loss fragment has not been covered with sufficient signal-to-noise ratio and resolution to allow for a quantitative analysis, and the slope change in the breakdown curve at $10.85 \mathrm{eV}$, also marked by the two straight lines, suggests a possibly complicated mechanism. The barrier to $\left(\mathrm{C}_{2} \mathrm{H}_{5} \mathrm{OH}_{2}\right)\left(\mathrm{OCH}_{2}\right)^{+}$further dissociating into formaldehyde and protonated ethanol is expected to lie around $10.6 \mathrm{eV}$, which may be responsible for the slope change along with, e.g., larger protonated ethanol clusters contributing to the protonated monomer signal by neutral ethanol loss. The contribution of the latter process could be measured by varying the molecular beam composition and the dimer/larger cluster ratio.

In modeling the breakdown diagram, the thermal energy distribution is calculated based on ab initio harmonic vibrational frequencies and rotational constants, taken from CBS-APNO calculations for the dimer, and using the same model for the monomer as previously. ${ }^{9}$ Juxtaposing experimental results with computational predictions can further our understanding of the fate of the internal energy selected parent ion. For example, very long direct dynamics calculations could be expected to reproduce the observed branching ratios without the need for a statistical model. However, because of the limited trajectory propagation times, such calculations can at most serve to establish the reaction coordinate in very high energy parent ions. ${ }^{44}$ Instead, the calculated dissociative
TABLE I. CBS-APNO calculated reaction energies of the ethanol dimer at $0 \mathrm{~K}$.

\begin{tabular}{lrc}
\hline \hline Reaction & \multicolumn{2}{c}{$\Delta E_{0 \mathrm{~K}} / \mathrm{eV}$} \\
\hline$\left(\mathrm{C}_{2} \mathrm{H}_{5} \mathrm{OH}\right)_{2} \rightarrow$ & $0.235^{\mathrm{a}}$ & \\
$2 \mathrm{C}_{2} \mathrm{H}_{5} \mathrm{OH}$ & 9.286 & \\
$\left(\mathrm{C}_{2} \mathrm{H}_{5} \mathrm{OH}\right)_{2}^{+}+e^{-}$ & 10.588 & \\
$\mathrm{C}_{2} \mathrm{H}_{5} \mathrm{OH}^{+}+\mathrm{C}_{2} \mathrm{H}_{5} \mathrm{OH}+e^{-}$ & 9.647 & \\
$\left(\mathrm{C}_{2} \mathrm{H}_{5} \mathrm{OH}_{2}\right)\left(\mathrm{OCHCH}_{3}\right)^{+}+\mathrm{H}+e^{-}$ & 9.723 & $9.76 \pm 0.05^{\mathrm{b}}$ \\
$\left(\mathrm{C}_{2} \mathrm{H}_{5} \mathrm{OH}_{2}\right)\left(\mathrm{OCH}_{2}\right)^{+}+\mathrm{CH}_{3}+e^{-}$ & & $9.719 \pm 0.004^{\mathrm{c}}$ \\
& 9.836 & \\
& $10.346^{\mathrm{d}}$ & $10.25 \pm 0.05^{\mathrm{b}}$ \\
$\left(\mathrm{C}_{2} \mathrm{H}_{5} \mathrm{OH}\right)\left(\mathrm{CH}_{2} \mathrm{OH}\right)^{+}+\mathrm{CH}_{3}+e^{-}$ & & \\
$\mathrm{C}_{2} \mathrm{H}_{5} \mathrm{OH}_{2}+\mathrm{C}_{2} \mathrm{H}_{5} \mathrm{O}+e^{-}$ & $1.374^{\mathrm{e}}$ & \\
\hline $\mathrm{C}_{2} \mathrm{H}_{5} \mathrm{OH}_{2} \mathrm{H}^{+} \rightarrow$ & \\
$\mathrm{C}_{2} \mathrm{H}_{5} \mathrm{OH}_{2}^{+}+\mathrm{C}_{2} \mathrm{H}_{5} \mathrm{OH}$ & \\
\hline \hline
\end{tabular}

a $22.6 \mathrm{~kJ} \mathrm{~mol}^{-1}$

${ }^{\mathrm{b}} \mathrm{Li}$ et al. ${ }^{14}$

${ }^{\mathrm{c}}$ Experimental value, this work.

${ }^{d}$ The CBS-APNO geometry optimization step did not converge for $\mathrm{C}_{2} \mathrm{H}_{5} \mathrm{O}$. The $\mathrm{C}_{2} \mathrm{H}_{5} \mathrm{O}$ CBS-APNO energy was derived based on the ethanol CBS-APNO energy and the W1U calculated $\mathrm{O}-\mathrm{H}$ bond energy in ethanol

${ }^{\mathrm{e}} 132.6 \mathrm{~kJ} \mathrm{~mol}^{-1}$ cf. the binding energy in the dimer cation to the monomer ion and neutral of $1.301 \mathrm{eV}, 125.5 \mathrm{~kJ} \mathrm{~mol}^{-1}$.

photoionization energetics can be completed with constrained geometry optimizations, transition state, and excited state calculations.

The measured methyl-loss dissociative photoionization onset connects the neutral ethanol dimer with the protonated ethanol-formaldehyde adduct, both of which have unknown enthalpies of formation. However, with the help of CBS-APNO calculated reaction energies, listed in Table I, a thermochemical cycle can be set up as shown in Figure 4(a) together with the neutral ethanol dimer and some of its ionic fragmentation products referenced in Table I. The $0 \mathrm{~K}$ enthalpy of formation of ethanol, ${ }^{45}$ proton $^{46}$ and the methyl radical, ${ }^{53}$ as well as formaldehyde ${ }^{47}$ has been reported at $-217.6 \pm 0.3,1528.084,150.0 \pm 0.3$, and $-105.42 \pm 0.33$ $\mathrm{kJ} \mathrm{mol}^{-1}$, respectively. Ion mobility measurements yielded a relative proton affinity of ethanol of $779.4 \pm 0.8 \mathrm{~kJ} \mathrm{~mol}^{-1}$ (a)

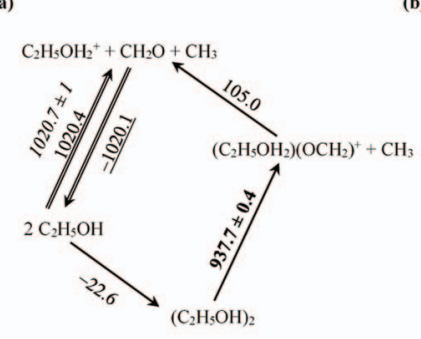

(b)

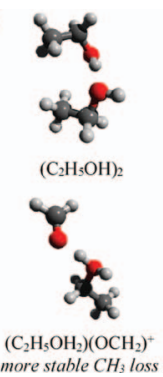

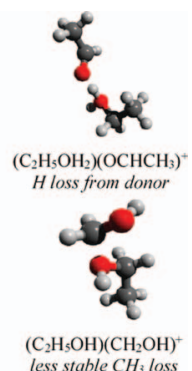

less stable $\mathrm{CH}_{3}$ loss
FIG. 4. A thermochemical cycle is shown in (a) starting from two ethanol molecules with the measured methyl-loss onset of the dimer in bold. The dimer binding energy and the binding energy of the protonated ethanol-formaldehyde cluster are calculated using the CBS-APNO composite method. The end products lie $1020.1 \mathrm{~kJ} \mathrm{~mol}^{-1}$ higher than the reactant ethanol. The products can also be reached directly, the computed reaction energy of $1020.4 \mathrm{~kJ} \mathrm{~mol}^{-1}$ compares well with the thermochemical cycle result as well as with $1020.7 \pm 1 \mathrm{~kJ} \mathrm{~mol}^{-1}$, based on published enthalpies of formatting, including a proposed revision ${ }^{49}$ of the ethanol proton affinity. The structure of the neutral dimer, and the $\mathrm{H}$-loss and $\mathrm{CH}_{3}$-loss products in dissociative photoionization are shown in (b). 
at room temperature. ${ }^{48}$ This value is revised by $-4.7 \mathrm{~kJ}$ $\mathrm{mol}^{-1}$ to $-774.7 \mathrm{~kJ} \mathrm{~mol}^{-1}$ in the NIST Chemistry Webbook based on a correction in ancillary thermochemical values. ${ }^{49}$ Based on W1U-calculated thermal enthalpies, the revised value converts to $769.6 \mathrm{~kJ} \mathrm{~mol}^{-1}$ at $0 \mathrm{~K}$, in good agreement with the W1U-and G4-calculated $0 \mathrm{~K}$ ethanol proton affinity, both at $770.9 \mathrm{~kJ} \mathrm{~mol}^{-1}$. Thus, the experimental closure of the thermochemical cycle shown in Figure 4 is calculated at $1020.7 \pm 1 \mathrm{~kJ} \mathrm{~mol}^{-1}$, in excellent agreement with the calculated 1020.4 and the mixed experimental/calculated reaction energy of $1020.1 \mathrm{~kJ} \mathrm{~mol}^{-1}$. The good agreement suggests that the computed reaction energies are probably quite accurate, but the only purely experimentally derived thermochemical conclusion is that the $0-\mathrm{K}$ binding energy in the protonated ethanol-formaldehyde cluster is $82.9 \pm 1.1 \mathrm{~kJ}$ $\mathrm{mol}^{-1}$ larger than that in the ethanol dimer.

Ethanol monomer cations lose an $\mathrm{H}$ atom in the lowest energy channel, which is followed at higher energies by $\beta-\mathrm{C}-$ $\mathrm{C}$ cleavage to yield protonated formaldehyde. Dimers never lose $\mathrm{H}$, the lowest energy channel is $\beta-\mathrm{C}-\mathrm{C}$ bond rupture instead, with the next channel being protonated monomer production by $\mathrm{C}_{2} \mathrm{H}_{5} \mathrm{O}$-loss. Larger than dimer cluster parent ions are never observed, they always lose at least a $\mathrm{C}_{2} \mathrm{H}_{5} \mathrm{O}$ radical to yield a protonated ethanol cluster. The Franck-Condon envelope for photoionization in higher clusters and the corresponding dissociation channels are not discussed here. Instead, the disappearance of the H-loss channel in the dimer ion is addressed. CBS-APNO calculated reaction energies for the dimer, listed in Table I, show that this change is counterintuitive based on energetics reasons alone. Even though $\mathrm{CH}_{3}$ loss is less energetic in the dimer because of the stabilization of the $\mathrm{OH}$ proton in the product protonated ethanol structure, it is still higher in energy than simple $\mathrm{H}$-loss from the $\mathrm{H}$-donor ethanol unit. Density functional theory (DFT) calculations are known to describe dispersion interactions poorly, and in the absence of experimental data, long-range dispersion corrected functionals are barely characterized for barriers in ionic systems. ${ }^{31,50-52}$ Therefore, we carried out a constrained $\mathrm{C}-\mathrm{H}$ bond length scan with second order perturbation theory, MP2/6-311++G(d,p), which indicated an H-loss reverse barrier, which is $100 \mathrm{meV}$ larger than in the ethanol monomer cation, i.e., about $250 \mathrm{meV}$. This reverse barrier lifts the effective barrier to H-loss to around $9.9 \mathrm{eV}$, which, together with the tighter transition state, ensures that H-loss from the dimer cation can never effectively compete with methyl loss via $\beta-\mathrm{C}-\mathrm{C}$ bond cleavage in the $\mathrm{H}$-donor ethanol unit. In short, the $\mathrm{CH}_{3}$-loss becomes more favored in the dimer because ethanol is protonated in the product instead of formaldehyde, as in the monomer, and ethanol has a higher proton affinity. It is because of this, and because of the increase in the reverse barrier to $\mathrm{H}$ loss that $\mathrm{H}$ loss is eliminated from the dissociative photoionization processes of the dimer.

Time-dependent DFT calculations were validated by EOM-IP-CCSD results for adiabatic ionization to the ground state and vertical ionization of the neutral. The average difference between EOM-IP-CCSD ionization energies with the cc-pVDZ and cc-pVTZ basis sets was about $0.3 \mathrm{eV}$. The adiabatic EOM-IP-CCSD dimer ionization energy is $8.685 \mathrm{eV}$ with the double- $\zeta$ basis set and $9.117 \mathrm{eV}$ with the triple- $\zeta$
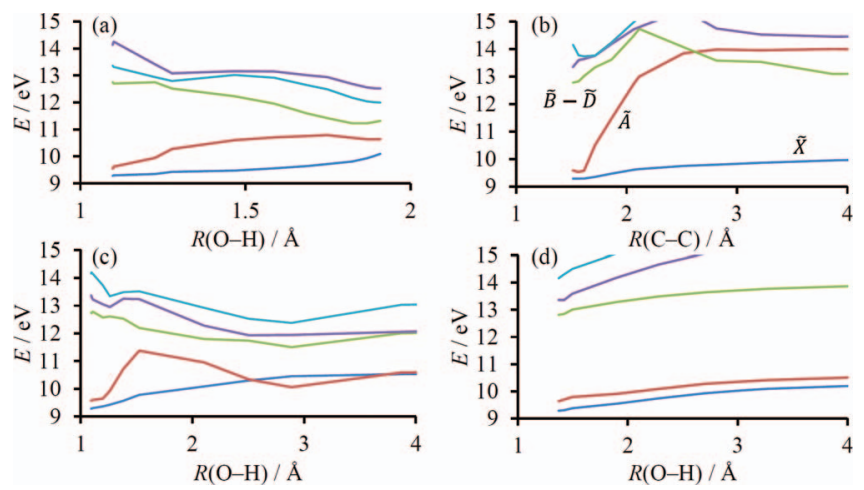

FIG. 5. M06-2X/aug-cc-pVTZ time dependent density functional theory results on the ethanol dimer ion, using the CBS-APNO dimer adiabatic ionization energy of $9.286 \mathrm{eV}$, for (a) relaxation of the vertically ionized ion by protonation of one ethanol subunit by the other, (b) $\mathrm{CH}_{3}$ loss by $\beta-\mathrm{C}-\mathrm{C}$ bond rupture in the $\mathrm{H}$-donor ethanol unit, (c) symmetric splitting of the dimer into $\mathrm{C}_{2} \mathrm{H}_{5} \mathrm{OH}^{+}$and $\mathrm{C}_{2} \mathrm{H}_{5} \mathrm{OH}$, and (d) asymmetric fragmentation into $\mathrm{C}_{2} \mathrm{H}_{5} \mathrm{OH}_{2}{ }^{+}$ $+\mathrm{C}_{2} \mathrm{H}_{5} \mathrm{O}$.

basis set. Since the agreement with the CBS-APNO result of $9.286 \mathrm{eV}$ is much better using the triple- $\zeta$ basis set, only ccpVTZ results are reported here. The vertical ionization energies of $9.883,10.979$, and $11.653 \mathrm{eV}$, as well as the ionization energies to the $\tilde{X}$ ion state minimum of $9.117,9.709$, and $13.069 \mathrm{eV}$ for the $\tilde{X}, \tilde{A}$, and $\tilde{B}$ states, respectively, show good qualitative agreement with the TD-DFT results with the M06-2X functional and aug-cc-pVTZ basis set as shown in Figure 5(a), in which the low and high $R(\mathrm{O}-\mathrm{H})$ limits corresponds to adiabatic and vertical ionization, respectively.

Li et al. ${ }^{14}$ suggest that $\mathrm{C}-\mathrm{C}$ bond breaking in the $\mathrm{H}$ acceptor yields $\left(\mathrm{C}_{2} \mathrm{H}_{5} \mathrm{OH}\right)\left(\mathrm{CH}_{2} \mathrm{OH}\right)^{+}$, i.e., the higher energy $\mathrm{CH}_{3}$-loss product (see Table I and Figure 4(b)) from the $\tilde{A}$ state of the dimer cation. Despite several attempts, no reaction path could be found corresponding to this process: $\mathrm{C}-\mathrm{O}$ bond contraction scans between the acceptor $\alpha-\mathrm{C}$ and the donor $\mathrm{O}$ actually yield the $\mathrm{H}$-loss product, and the $\mathrm{C}-\mathrm{O}$ bond is not formed in $\mathrm{C}-\mathrm{C}$ bond elongation scans. However, a few geometry optimizations seem to suggest that this process could nevertheless take place without a reverse barrier. Still, the breakdown diagram in Figure 3(b) does not indicate a new $\mathrm{CH}_{3}-$ loss channel opening up at $11 \mathrm{eV}$. Furthermore, the $\mathrm{CH}_{3}$-loss fragment ions may have up to $1.3 \mathrm{eV}$ internal energy around $11 \mathrm{eV}$ photon energy, which makes their isomerization readily possible. Thus, there appears to be no reason to correlate $\tilde{A}$ state dimer ions with a new $\mathrm{CH}_{3}$-loss fragment ion isomer. As far as $\mathrm{CH}_{3}$-loss from the donor is concerned, it is clear from Figure 5(b) that only the ground electronic state of the cation corresponds with the ground state products. As mentioned earlier, the complete disappearance of the $\mathrm{C}_{2} \mathrm{H}_{5} \mathrm{OH}^{+}$parent ion above the $\mathrm{H}$-loss threshold in the monomer breakdown diagram (Figure 3(a)) suggests that dimers and larger clusters never dissociate into $\mathrm{C}_{2} \mathrm{H}_{5} \mathrm{OH}^{+}$. The TD-DFT results in Figure 5(c) also rule out $\tilde{A}$ state participation, because symmetric fragmentation of the dimer into two ethanol units appears to go over a reverse barrier in the $\tilde{A}$ state of the dimer ion. Instead, the $242 \mathrm{meV}\left(23 \mathrm{~kJ} \mathrm{~mol}^{-1}\right)$ difference between the product energies and the tighter transition for symmetric bond breaking, which has to involve $\mathrm{H}$-transfer back to 
the leaving ethanol, mean that asymmetric bond breaking, as shown in Figure 5(d) and yielding $\mathrm{C}_{2} \mathrm{H}_{5} \mathrm{OH}_{2}{ }^{+}+\mathrm{C}_{2} \mathrm{H}_{5} \mathrm{O}$ always outcompetes symmetric bond breaking within the experimental detection limits. The $\tilde{B}$ state is completely decoupled from these dissociative photoionization processes at threshold, because it correlates adiabatically with excited state products.

In short, the energy diagram as proposed by Li et al. ${ }^{14}$ needs a few corrections. The HOMO-1 ionization yielding $\tilde{A}$ state ions does not yield measurable ethanol cation signal, and does not appear to open up a new methyl-loss channel, either, and rather follows the dissociative photoionization scheme established by the ground electronic state. The HOMO-2 ionization produces $\tilde{B}$ state ions, which must internally convert to the ground electronic state before fragmenting into ground state products. Therefore, there is no experimental or computational evidence suggesting that the initial ionization site or the photoion state plays a role in the subsequent fragmentation processes.

\section{CONCLUSIONS}

The dissociation of internal energy selected ethanol monomer and dimer cations was studied by photoelectron photoion coincidence. In the monomer breakdown diagram, the branching ratio between $\mathrm{H}$-loss and $\mathrm{CH}_{3}$-loss channels changes smoothly in the 11.1-11.5 eV photon energy range. The SSACM rate theory predicts oscillations in the branching ratio because of the tightness of the $\mathrm{CH}_{2} \mathrm{O}^{+}+\mathrm{CH}_{3}$ products and the resulting slope changes in the product number of states at each new vibrational state. Such oscillations were, however, not observed. Statistical dissociation rate models with few adjustable parameters are needed to model experimental rate curves. Neither of the two widely used theories, RRKM or SSACM yields a satisfactory model in the energy range slightly above the crossover of the $\mathrm{H}$-loss and $\mathrm{CH}_{3}$-loss signals.

The dimer ion loses a methyl group from the H-donor subunit in the lowest energy channel, the $0 \mathrm{~K}$ appearance energy for which has been measured at $9.719 \pm 0.004 \mathrm{eV}$. This value is used in a thermochemical cycle, which confirms CBS-APNO reaction energies and previously published thermochemical data. The experimental data as well as excited state calculations indicate that the dimer cation dissociates statistically below a photon energy of $12 \mathrm{eV}$, i.e., the initial electronic state plays no role in the dissociative photoionization process and the ergodic hypothesis holds. In such cases, the ionization process itself may be site or state specific, but the internal degrees of freedom are completely scrambled before the parent ion dissociates, and the dissociation mechanism is not a function of the intermediate ion state(s).

\section{ACKNOWLEDGMENTS}

The experimental work was carried out at the VUV beamline of the Swiss Light Source and the calculations were performed at the Merlin 4 HPC cluster at the Paul Scherrer Institut.
${ }^{1}$ T. A. Cool, K. Nakajima, T. A. Mostefaoui, F. Qi, A. Mcllroy, P. R. Westmoreland, M. E. Law, L. Poisson, D. S. Peterka, and M. Ahmed, J. Chem. Phys. 119, 8356 (2003).

${ }^{2}$ B. Ruscic and J. Berkowitz, J. Chem. Phys. 101, 10936 (1994).

${ }^{3}$ Z. A. Harvey and J. C. Traeger, J. Mass Spectrom. 39, 802 (2004).

${ }^{4}$ B. Ruscic, Res. Adv. Phys. Chem. 1, 39 (2000).

${ }^{5}$ T. Baer, B. Sztaray, J. P. Kercher, A. F. Lago, A. Bodi, C. Skull, and D. Palathinkal, Phys. Chem. Chem. Phys. 7, 1507 (2005).

${ }^{6}$ B. Sztáray, A. Bodi, and T. Baer, J. Mass Spectrom. 45, 1233 (2010).

${ }^{7}$ N. S. Shuman, M. Johnson, W. R. Stevens, M. E. Harding, J. F. Stanton, and T. Baer, J. Phys. Chem. A 114, 10016 (2010).

${ }^{8}$ S. Borkar, B. Sztáray, and A. Bodi, Phys. Chem. Chem. Phys. 13, 13009 (2011).

${ }^{9}$ A. Bodi, M. Daniel Brannock, B. Sztáray, and T. Baer, Phys. Chem. Chem. Phys. 14, 16047 (2012).

${ }^{10}$ W. Stevens, B. Sztaray, N. S. Shuman, T. Baer, and J. Troe, J. Phys. Chem. A 113, 573 (2009).

${ }^{11}$ J. Troe, V. G. Ushakov, and A. A. Viggiano, J. Phys. Chem. A 110, 1491 (2006).

${ }^{12}$ J. C. Light, J. Chem. Phys. 40, 3221 (1964).

${ }^{13}$ C. E. Klots, J. Chem. Phys. 64, 4269 (1976).

${ }^{14}$ W. Li, Y. Hu, J. Guan, F. Liu, X. Shan, and L. Sheng, J. Chem. Phys. 139, 024307 (2013).

${ }^{15}$ K.-M. Weitzel, G. Jarvis, M. Malow, T. Baer, Y. Song, and C. Ng, Phys. Rev. Lett. 86, 3526 (2001)

${ }^{16}$ A. Bodi, N. S. Shuman, and T. Baer, Phys. Chem. Chem. Phys. 11, 11013 (2009).

${ }^{17}$ T. Baer, A. Guerrero, J. Z. Davalos, and A. Bodi, Phys. Chem. Chem. Phys. 13, 17791 (2011).

${ }^{18}$ R. G. Palmer, Adv. Phys. 31, 669 (1982).

${ }^{19}$ J. Harvey, A. Bodi, R. P. Tuckett, and B. Sztaray, Phys. Chem. Chem. Phys. 14, 3935 (2012).

${ }^{20}$ J. Harvey, P. Hemberger, A. Bodi, and R. P. Tuckett, J. Chem. Phys. 138, 124301 (2013)

${ }^{21}$ I. G. Simm, C. J. Danby, J. H. D. Eland, and P. I. Mansell, J. Chem. Soc., Faraday Trans. 2 72, 426 (1976).

${ }^{22}$ J. C. Creasey, H. M. Jones, D. M. Smith, R. P. Tuckett, P. A. Hatherly, K. Codling, and I. Powis, Chem. Phys. 174, 441 (1993).

${ }^{23}$ R. J. Bemish, P. A. Block, L. G. Pedersen, W. Yang, and R. E. Miller, J. Chem. Phys. 99, 8585 (1993).

${ }^{24}$ D. C. Dayton, L. G. Pedersen, and R. E. Miller, J. Chem. Phys. 93, 4560 (1990).

${ }^{25}$ T. Baer and A. R. Potts, J. Phys. Chem. A 104, 9397 (2000).

${ }^{26}$ U. Lourderaj and W. L. Hase, J. Phys. Chem. A 113, 2236 (2009).

${ }^{27}$ A. Bodi, M. Johnson, T. Gerber, Z. Gengeliczki, B. Sztaray, and T. Baer, Rev. Sci. Instrum. 80, 034101 (2009).

${ }^{28}$ M. Johnson, A. Bodi, L. Schulz, and T. Gerber, Nucl. Instrum. Methods Phys. Res. A 610, 597 (2009).

${ }^{29}$ A. Bodi, P. Hemberger, T. Gerber, and B. Sztáray, Rev. Sci. Instrum. 83, 083105 (2012).

${ }^{30}$ B. Sztaray and T. Baer, Rev. Sci. Instrum. 74, 3763 (2003).

${ }^{31}$ A. Bodi, B. Sztaray, and T. Baer, Phys. Chem. Chem. Phys. 8, 613 (2006).

${ }^{32}$ B. West, C. Joblin, V. Blanchet, A. Bodi, B. Sztáray, and P. M. Mayer, J. Phys. Chem. A 116, 10999 (2012).

${ }^{33}$ J. W. Ochterski, G. A. Petersson, and J. A. Montgomery, J. Chem. Phys. 104, 2598 (1996).

${ }^{34}$ Y. Zhao and D. G. Truhlar, Theor. Chim. Acta 120, 215 (2008).

${ }^{35}$ M. J. Frisch, G. W. Trucks, H. B. Schlegel et al., Gaussian 09, Revision A.1 Gaussian, Inc., Wallingford, CT, 2009.

${ }^{36}$ E. C. Barnes, G. A. Petersson, J. A. Montgomery, M. J. Frisch, and J. M. L. Martin, J. Chem. Theory Comput. 5, 2687 (2009).

${ }^{37}$ D. Sinha, S. K. Mukhopadhyay, R. Chaudhuri, and D. Mukherjee, Chem. Phys. Lett. 154, 544 (1989).

${ }^{38}$ C. W. Murray and E. R. Davidson, Chem. Phys. Lett. 190, 231 (1992).

${ }^{39}$ Y. Shao, L. F. Molnar, Y. Jung, J. Kussmann, C. Ochsenfeld, S. T. Brown, A. T. B. Gilbert, L. V. Slipchenko, S. V. Levchenko, D. P. O'Neill, R. A. DiStasio, R. C. Lochan, T. Wang, G. J. O. Beran, N. A. Besley, J. M. Herbert, C. Y. Lin, T. Van Voorhis, S. H. Chien, A. Sodt, R. P. Steele, V. A. Rassolov, P. E. Maslen, P. P. Korambath, R. D. Adamson, B. Austin, J. Baker, E. F. C. Byrd, H. Dachsel, R. J. Doerksen, A. Dreuw, B. D. Dunietz, A. D. Dutoi, T. R. Furlani, S. R. Gwaltney, A. Heyden, S. Hirata, C.P. Hsu, G. Kedziora, R. Z. Khalliulin, P. Klunzinger, A. M. Lee, M. S. Lee, W. Liang, I. Lotan, N. Nair, B. Peters, E. I. Proynov, P. A. Pieniazek, Y. M. Rhee, J. Ritchie, E. Rosta, C. D. Sherrill, A. C. Simmonett, 
J. E. Subotnik, H. L. Woodcock, W. Zhang, A. T. Bell, A. K. Chakraborty, D. M. Chipman, F. J. Keil, A. Warshel, W. J. Hehre, H. F. Schaefer, J. Kong, A. I. Krylov, P. M. W. Gill, and M. Head-Gordon, Phys. Chem. Chem. Phys. 8, 3172 (2006).

${ }^{40}$ A. I. Krylov and P. M. W. Gill, Wiley Interdiscip. Rev.: Comput. Mol. Sci. 3, 317 (2013).

${ }^{41}$ B. L. Yoder, A. H. C. West, B. Schläppi, E. Chasovskikh, and R. Signorell, J. Chem. Phys. 138, 044202 (2013).

${ }^{42}$ A. Bodi, W. R. Stevens, and T. Baer, J. Phys. Chem. A 115, 726 (2011).

${ }^{43}$ W. R. Stevens, A. Bodi, and T. Baer, J. Phys. Chem. A 114, 11285 (2010).

${ }^{44}$ P. M. Mayer, D. Staedter, V. Blanchet, P. Hemberger, and A. Bodi, J. Phys. Chem. A 117, 2753 (2013).

${ }^{45} \mathrm{E}$. Goos, A. Burcat, and B. Ruscic, Extended Third Millennium Ideal Gas and Condensed Phase Thermochemical Database for Combustion With Updates From Active Thermochemical Tables (2012), available at http://garfield.chem.elte.hu/Burcat/burcat.html (retrieved on 3 October 2013).
${ }^{46}$ B. Ruscic, Active Thermochemical Tables Early Beta 1.110 (retrieved on 14 July 2011), see http://atct.anl.gov/Thermochemical\%20Data/ version\%20earlyBeta\%201.110/index.html.

${ }^{47}$ G. Czakó, B. Nagy, G. Tasi, Á. Somogyi, J. Šimunek, J. Noga, B. J. Braams, J. M. Bowman, and A. G. Császár, Int. J. Quantum Chem. 109, 2393 (2009).

${ }^{48}$ M. Tabrizchi and S. Shooshtari, J. Chem. Thermodyn. 35, 863 (2003).

${ }^{49}$ NIST Chemistry WebBook, NIST Standard Reference Database Number 69 , edited by W. G. Mallard and P. J. Linstrom (National Institute of Standards and Technology, Gaithersburg, MD, 2013).

${ }^{50}$ S. Tsuzuki and H. P. Lüthi, J. Chem. Phys. 114, 3949 (2001).

${ }^{51}$ P. Hemberger, A. Bodi, C. Schon, M. Steinbauer, K. H. Fischer, C. Kaiser, and I. Fischer, Phys. Chem. Chem. Phys. 14, 11920 (2012).

${ }^{52}$ S. Grimme, J. Comput. Chem. 27, 1787 (2006).

${ }^{53}$ B. Ruscic, J. E. Boggs, A. Burcat, A. G. Csaszar, J. Demaison, R. Janoschek, J. M. L. Martin, M. L. Morton, M. J. Rossi, J. F. Stanton, P. G. Szalay, P. R. Westmoreland, F. Zabel, and T. Berces, J. Phys. Chem. Ref. Data 34, 573 (2005). 